\title{
Direct comparison of elastic incoherent neutron scattering experiments with molecular dynamics simulations of DMPC phase transitions
}

Bachir Aoun $^{a, \ddagger}$, Eric Pellegrini $^{a}$, Marcus Trapp $^{\mathrm{b}, \mathrm{c}}$, Francesca Natali $^{\mathrm{a}, \mathrm{d}}$, Laura Cantu’ ${ }^{\mathrm{e}}$, Paola

Brocca $^{\mathrm{e}}$, Yuri Gerelli ${ }^{\mathrm{a}}$, Bruno Demé ${ }^{\mathrm{a}}$, Michael Marek Koza ${ }^{\mathrm{a}}$, Mark Johnson ${ }^{\mathrm{a}}$, and Judith

Peters $^{\mathrm{a}, \mathrm{f}_{*}}$

\footnotetext{
${ }^{\text {a }}$ Institut Laue-Langevin, 71 avenue des Martyrs, CS 20156, 38042 Grenoble Cedex 9, France

b Angewandte Physikalische Chemie, Universität Heidelberg, Im Neuenheimer Feld 253, 69120 Heidelberg, Germany

${ }^{\mathrm{c}}$ Helmholtz-Zentrum Berlin für Materialien und Energie, Lise-Meitner Campus, Hahn-Meitner-Platz 1, 14109 Berlin, Germany

${ }^{\mathrm{d}}$ CNR-IOM-OGG, c/o Institut Laue-Langevin, 71 avenue des Martyrs, CS 20156, 38042 Grenoble Cedex 9, France

${ }^{\mathrm{e}}$ University of Milan, via F. 1li Cervi 93,20090 Segrate, Italy

${ }^{\mathrm{f}}$ Univ. Grenoble Alpes, IBS and LiPhy, UFR PhITEM,71 avenue des Martyrs, CS 10090 , 38044 Grenoble, France
} 


\section{Abstract}

Neutron scattering techniques have been employed to investigate 1,2-dimyristoyl-sn-glycero3-phosphocholine (DMPC) membranes in the form of multilamellar vesicles (MLVs) and deposited, stacked multilamellar-bilayers (MLBs), covering transitions from the gel to the liquid phase. Neutron diffraction was used to characterise the samples in terms of transition temperatures, whereas elastic incoherent neutron scattering (EINS) demonstrates that the dynamics on the sub-macromolecular length-scale and pico- to nano-second time-scale are correlated with the structural transitions through a discontinuity in the observed elastic intensities and the derived mean square displacements. Molecular dynamics simulations have been performed in parallel focussing on the length-, time- and temperature-scales of the neutron experiments. They correctly reproduce the structural features of the main gel-liquid phase transition. Particular emphasis is placed on the dynamical amplitudes derived from experiment and simulations. Two methods are used to analyse the experimental data and mean square displacements and they agree within a factor of 2. Mean square displacements obtained directly from simulations show a comparable level of agreement with experimental values. Experiments and simulations together give therefore a consistent picture of the structural and dynamical aspects of the main lipid transition and provide a basis for future, theoretical modelling of dynamics and phase behaviour in membranes. The need for analytical models is highlighted by the remaining discrepancy in dynamical amplitudes between experiment and simulation which, together with the significant differences between amplitudes derived in different ways from experimental data, points to the need for a systematic study of a wide range of systems. 
A detailed understanding of lipid systems is of paramount importance in biology, as each type of cellular membrane contains its own lipid composition, which is characterized by its chemical nature, chain length and degree of saturation. Cell membranes can in fact be composed of more than a hundred different types of lipids. They are not only structural elements, but also have a key role for the specificity of biological properties of the membrane. For a more complete overview, see the textbook of O. Mouritsen [1].

Although 1,2-dimyristoyl-sn-glycero-3-phosphocholine (DMPC) is among the most studied lipid systems, both experimentally and by molecular dynamics (MD) simulations, direct comparisons between the corresponding data, which entail performing the simulations in conditions matching those of the experiments, are under-represented. It is well-established by a number of groups that atomistic simulations can accurately reproduce a range of, typically structural, membrane properties [2, 3, 4] with microscopic dynamics being less extensively investigated and validated. Some relevant examples can, however, be found in $[5,6,7]$. Experimentally, mainly the mechanisms behind lipid phase transitions have been investigated using methods such as calorimetry [8], electron paramagnetic resonance [9], dilatometry [10, 11, 12], light transmittance [13, 14], Fourier transform infrared spectroscopy (FTIR) [15] and small angle scattering of neutrons (SANS) or X-rays (SAXS) [16, 17]. Neutron and X-ray diffraction [18], spectroscopy [19], reflectometry [20] and solid-state NMR [21] have also given information on the structure and dynamics of model lipid membranes.

The complexity of working both experimentally and with simulations on membranes may explain in part why these complementary approaches have not been brought together more often in the past. In this work, we have therefore combined calorimetry and neutron scattering experimental techniques with atomistic simulations using the NAMD-2.6 program [22] and the CHARMM36 force field [23] to develop a quantitative comparison between the experimental and computational models of lipid membrane structure and, in particular, dynamics. 
A recent survey of neutron scattering, MD and theoretical studies of bio-molecular dynamics revealed about 50 papers based on simulations, $60 \%$ of which were focused on 4 proteins [24]. About 15 of the 50 papers made direct comparison between experimental and simulation data although, as a rule, both types of work were performed and published independently and similarity in trends between experimental and simulation results were sought. Given the increasing availability and use of $\mathrm{MD}$, it is timely to perform a careful, direct comparison of experiment and simulation, especially for the dynamics of a reference, membrane system DMPC.

In this work we have concentrated on multilamellar vesicles (MLVs) and multilamellar bilayers (MLBs), see figure 1, in membrane systems made of DMPC, which are often used to mimic their more complex, natural counterparts as cell membranes because of their similar thermodynamical behavior. As a function of molecular geometry, temperature, concentration and hydration, the lipids self-assemble in different phases, such as micelles, MLVs, lamellar, cubic or hexagonal phases. Whereas MLVs are usually prepared with an excess of water, laterally ordered systems can occur in different hydration states going from completely dry to fully hydrated. It is very difficult to reach full hydration, as it depends sensitively on the exact temperature and sample surface area [25] and on how the sample is prepared and conserved during the experiment to avoid uncontrolled water exchange between the sample and the environment.

Focusing on bilayer-type structures, the commonly observed fully hydrated, lamellar states are (see figure 2):

a) $L_{c}$ phase: the lamellar crystalline phase is the most ordered one at low temperature. In this phase, lateral lipid diffusion is mostly absent.

b) $\mathrm{L}_{\beta}$, phase: In this so called 'gel' phase, the lipid chains are ordered in the all-trans configuration. The prime indicates that the alkyl chains are tilted with respect to the bilayer normal. In the case of DMPC, an angle of about $30^{\circ}$ is observed (the exact value is temperature dependent). The high degree of chain order leads to a quasi-crystalline order of lipids within the individual lamellae.

c) $\mathrm{P}_{\beta}$, phase: the 'ripple' phase. This phase does not exist in all phospholipids. If existing, it is formed prior to the main phase transition. It exhibits one dimensional ripples on the membrane surface. Close to the main phase transition, a co-existence of rafts of $\mathrm{L}_{\alpha}$ domains in 
a $\mathrm{P}_{\beta}$, continuum and of $\mathrm{P}_{\beta}$, domains in an $\mathrm{L}_{\alpha}$ continuum with increasing temperature was observed [26].

d) $\mathrm{L}_{\alpha}$ phase: the liquid-disordered or fluid phase. The long-range in-plane order is lost. This corresponds largely to the physiological state in cells.

The various types of lipids have different main phase transition temperatures $T_{m}$ (between the gel and the fluid phases), depending on the interactions between lipids and thus on their chain lengths, saturation, polar head group charges, etc. Some of their characteristics can be found in $[8,27,28]$. If the external conditions (salinity, $\mathrm{pH}$, hydration, pressure or temperature) require it, the cells have the capacity to adapt the lipidic composition of their membrane by a metabolic response. Such processes lower considerably the phase transition temperature and help the organisms to survive, if necessary. On the contrary, low hydration provokes an increase of $\mathrm{T}_{\mathrm{m}}[29]$.

The exact temperature of the phase transition strongly depends on the mesoscopic shape of the aggregate [30, 31], that is on its curvature. It is generally believed that the phase behavior is the result of a competition between the elastic free energy, depending on the intrinsic radius of curvature when packing many lipids together, and the resulting hydrocarbon packing strains [8]. Curvature is clearly coupled with particle size and with the degree of hydration.

Core fluidity is an important concept in membranes with respect to their mechanical properties as it allows for overall membrane deformation from the average shape and diffusion of embedded components within the membrane itself. The lipids (and proteins) can undergo different motions within the membrane, from simple vibrations, rotations and translations up to exchanges from one layer to the other (flip-flop), all of them corresponding to characteristic time-scales [32]. An experimental technique to observe short range lipid dynamics and phase transitions at time-scales on the order of pico-nanoseconds (ps-ns) is elastic incoherent neutron scattering (EINS). Neutron wavelengths ( $\AA$ to $\mathrm{nm}$ ) span interatomic and inter-molecular distances making neutron spectroscopy sensitive to motional amplitudes in macromolecules. In particular, incoherent neutron scattering provides detailed information on the motion of the Hydrogen $(\mathrm{H})$ atoms present in the sample as their incoherent scattering cross section exceeds largely those of all other atoms present in biological samples. Motions occurring within the ps-ns time window are of particular interest 
since they cover the crossover region from local excitations to slower processes involving collective motions of the whole membrane, like bending.

However, despite the investigations by neutron scattering and nuclear magnetic resonance (NMR) of S. König et al. published in 1992 [33] and 1994 [34], and many efforts to elucidate different aspects of lipid movements [see, for instance, 35, 36, 37, 38], a complete theoretical model taking into account all possible lipid motions, and a theory explaining lipid phase transitions, does not yet exist. It could, for example, provide a model for the motions of head groups and tails of lipids giving a relevant expression for the elastic incoherent structure factor (EISF). In this context, MD simulations become an essential source of accurate microscopic details on such systems, provided that they closely reproduce the experimental data.

We have thus measured structure and H-diffusion in DMPC MLVs and MLBs on diffractometers and spectrometers at the Institut Laue Langevin (ILL), Grenoble, France. Diffraction and calorimetry were used to characterize the samples prior to spectroscopic measurements. We have focused on the correlation between structural and, particularly, dynamical aspects of the phase transitions and how well they are reproduced by simulations performed, as far as possible, under the same conditions. Correlation functions calculated from MD simulations highlight the known limitations of the Gaussian approximation which is widely-used to obtain the mean square displacement from experimental data. For this reason, a second method, which uses the full range of experimental data, has been used to determine dynamical amplitudes.

\section{Experimental details}

\section{Sample preparation}

For all sample preparations, DMPC was purchased either from Lipoid (Ludwigshafen, Germany) or from Avanti Polar Lipids (Alabaster, USA) and used without further purification.

To produce DMPC MLVs, about $100 \mathrm{mg}$ of lipid powder were placed in a flat sample holder and hydrated in a desiccator from pure $\mathrm{D}_{2} \mathrm{O}$ for two days at $40^{\circ} \mathrm{C}$. Additional heavy water was added to achieve a sample with an excess of water [36]. In order to verify that the sample 
preparation reproduces the well-known transition temperatures observed in MLVs, for the pre-transition at $13^{\circ} \mathrm{C}\left(286 \mathrm{~K}-\mathrm{T}_{\mathrm{p}}\right)$ and for the main phase transition at $24^{\circ} \mathrm{C}\left(297 \mathrm{~K}-\mathrm{T}_{\mathrm{m}}\right)$, differential scanning calorimetry (DSC, MicroDSC Setaram Instrumentation (Caluire, France)) experiments were carried out prior to the neutron scattering experiments. The DSC measurement on the MLV sample was performed on cooling at a rate of 24 degrees per hour.

DMPC highly-oriented, MLBs were prepared on Si wafers and hydrated with heavy water. We used the "rock and roll" method following a protocol described by Tristram-Nagle and co-workers [39] in which DMPC powder was deposited on a $\mathrm{Si}(111)$ wafer of dimensions $30 \mathrm{x} 40 \mathrm{x} 0.38 \mathrm{~mm}^{3}$ by evaporating from a trifluoroethanol:chloroform mixture $(2: 1, \mathrm{v} / \mathrm{v})$. After deposition, the wafer was dried over silica gel for 2 days in a desiccator. The sample was rehydrated from pure $\mathrm{D}_{2} \mathrm{O}$ at $40{ }^{\circ} \mathrm{C}$ to achieve a high hydration level. Hereafter, the hydrated state refers to at least 14 water molecules per lipid whereas the dry state means about 2 water molecules per lipid. One wafer contained a total amount of $\approx 35 \mathrm{mg}$ of lipids.

Both MLVs and oriented, MLB samples were then placed in slab-shaped aluminum sample holders, gold-coated to prevent sample contamination. Sample cells were sealed using indium wire and the weight of the sample was monitored before and after the experiment, with no change observed indicating a stable level of hydration.

\section{Neutron scattering experiments}

The sample structures were first characterized at the small momentum transfer diffractometer D16 [40] (ILL) by a $\theta-2 \theta$ scan, with incident neutron wavelength $\lambda=4.75 \AA$. The diffracted neutrons were recorded on a two-dimensional, area detector. From the angle $\theta$, the modulus of the wave vector transfer Q can be calculated through

$$
Q=\frac{4 \pi}{\lambda} \sin (\theta),
$$

and the corresponding d-spacing of the lamellae is

$$
d=\frac{2 \pi}{Q}
$$


The lamellar spacing found for close to fully hydrated DMPC samples can vary between 50 and $62.7 \AA$ [41], depending on temperature and the exact sample preparation conditions. The mosaic spread of MLB's was determined from a rocking scan in which the sample is rotated with respect to the incoming beam ( $\omega$-axis) to cover the full width at half maximum (FWHM) of the first order (001) Bragg reflection. The mosaic spread is an indication of membrane order relative to the mean orientation of the membrane stack. A typical value of the mosaïcity for solid supported membranes is $\sim 1^{\circ}$ or less over several thousands of bilayers and was confirmed here.

For dynamics investigations, EINS temperature scans were performed on the thermal neutron, backscattering spectrometer IN13 [42] in the case of MLVs and on the cold neutron, time-offlight spectrometer IN6 [43] in the case of MLBs. The precision of temperature determination is about $\pm 1 \mathrm{~K}$ for both instruments. IN13 gives access to high momentum transfers $(0.2<\mathrm{Q}<$ $4.9 \AA^{-1}$ ) with an almost Q-independent energy resolution of $8 \mu \mathrm{eV}$ (FWHM), due to the incident wavelength of $\lambda=2.23 \AA$. On IN6, the incident wavelength $\lambda$ of $5.1 \AA$ gives an elastic energy resolution of $90 \mu \mathrm{eV}(\mathrm{FWHM})$ and momentum transfer range of $0.44<\mathrm{Q}<2.0$ $\AA^{-1}$. The corresponding, accessible time and length-scales on the spectrometers are given in table 1.

The elastic scattering intensities $\left(\mathrm{I}_{\mathrm{el}}=\mathrm{S}(\mathrm{Q}, \omega \approx 0)\right)$ were corrected for the empty cell (+ clean wafer contributions for the membranes) subtracting the corresponding measurement from the sample intensity. They were further normalized with respect to vanadium, a dominantly incoherent scatterer, to correct for detector efficiency variations. In order to avoid multiple scattering, the sample thickness was calculated to give a transmission of about $\sim 90 \%$.

Atomic mean square displacements (MSD) $\left\langle u^{2}\right\rangle$ were extracted from the elastic intensities through

$$
\ln I_{e l} \approx-\frac{1}{6}\left\langle u^{2}\right\rangle Q^{2}
$$

in the low Q-range, where the Gaussian approximation [44] is valid [45]. They represent the harmonic motions of the atoms around their equilibrium positions and reflect the global flexibility of a sample. The phase transition of the lipids is seen as a pronounced change in the temperature dependent behavior of the elastic intensity as the lipids enter the fluid phase. On 
IN13 the Q-range of 0.5 to $1.45 \AA^{-1}$ was chosen to extract the MSD in the Gaussian approximation $\left(\mathrm{MSD}_{\mathrm{GA}}\right)$ (see Electronic Supplementary Information, figure 1). Summed elastic intensities reveal directly the phase transition and they were calculated taking into account the whole Q-range available on IN13: $0.3 \AA^{-1}<\mathrm{Q}<4.9 \AA^{-1}$. On IN6 we used the elastic intensities summed over all Q-values up to $1.3 \AA^{-1}$, to exclude the coherent chain correlation peak for MLB's around $1.48 \AA^{-1}$. To determine the $\mathrm{MSD}_{\mathrm{GA}}$ from the IN6 data, Q values up to $0.69 \AA^{-1}$ were used. The measurements on oriented membranes were performed with the sample at an angle of $135^{\circ}$ with respect to the incoming beam, where the momentum transfer $\mathrm{Q}$ is predominantly oriented parallel to the membrane surface to detect in-plane membrane dynamics [46].

The Gaussian approximation restricts to low $\mathrm{Q}$, in which the linearity between $\ln \left(\mathrm{I}_{\mathrm{el}}\right)$ and $\mathrm{Q}^{2}$ in equation 3 is satisfied, but the experimental data that can be used to determine the MSD and the result can depend sensitively on the selected Q-range. We have therefore also used a non-Gaussian model, presented in [47], to extract mean square atomic position fluctuations (MSPF) from the whole $\mathrm{Q}$ range. Thus two experimental determinations of the dynamical amplitudes can be compared with the MSD from the MD simulations, in all cases taking into account the energy resolution of the spectrometers.

Heating rates for neutron measurements were determined to a large extent by the measurement time per point on the corresponding instrument. On D16, the temperature was increased by 3 degrees per hour. On IN13, each temperature point was measured for 10 hours with an additional hour for equilibration - the corresponding heating rate was about 3 hours per degree. On IN6, the heating rates were 24 degrees per hour from 240 to $280 \mathrm{~K}$ and 9 degrees per hour from 280 to $340 \mathrm{~K}$, because neutron intensity decreases with rising temperature. These heating rates were based on previous experiments and there was no evidence of hysteresis in the measurements or that the samples did not reach equilibrium at each temperature.

\section{Molecular dynamics simulations}

The NAMD-2.6 program [22] with the CHARMM36 force field [23], including the TIP3P model for water, was used in this work. Three lipid bilayer models containing 72 DMPC 
molecules (36 lipids per leaflet), with different hydration levels of 2, 12 and 25 molecules of water per lipid, thus spanning from dry to full hydration, were prepared using the CHARMMGUI membrane builder [48]. MD simulation production runs of equilibrated systems were performed at 8 different temperatures ( $280 \mathrm{~K}$ to $350 \mathrm{~K}$ in $10 \mathrm{~K}$ steps), covering the expected gel-liquid transition temperature around $300 \mathrm{~K}$ and higher temperatures at which the gel-liquid transition is sometimes observed in lamellar membranes by EINS. In addition, the wide temperature range is well-adapted for the dry membrane. The size and contents of the simulation box were chosen to cover the dynamical length-scale and with consideration for the large number of simulations to be performed here: 24 runs exceeding a total simulation time of $2.4 \mu \mathrm{s}$. This simulation box is similar to that used in other work [49], but is smaller than that in more recent work which focused on only two temperatures [50].

The simulation protocol consisted of two distinct steps used to bring the initial temperature state to $280 \mathrm{~K}$. First, $10^{4}$ steps of energy minimization using the conjugate gradient method were performed in order to remove any close contacts from the starting structure. In the second step, the system was progressively heated from $0 \mathrm{~K}$ to $280 \mathrm{~K}$ by steps of $20 \mathrm{~K}$ in order to smoothly bring the system to the target temperature. Each heating step was performed using the NVT ensemble for 10ps with a time step of 1fs. Langevin dynamics was used to control the temperature.

Thereafter, each of the three differently hydrated systems underwent equilibration and production runs. The equilibration was performed using the isothermal - isobaric NPT ensemble over $100 \mathrm{~ns}$ with a time step of $2 \mathrm{fs}$. Langevin dynamics and the Langevin piston method were used to control the temperature and pressure. The production run was performed in the NPT ensemble over $5 \mathrm{~ns}$ with a time step of $1 \mathrm{fs}$. In all the simulations, the non-bonded interactions were cut off at $12 \AA$, with a smooth switching function from $10 \AA$, and long-range electrostatic interactions were computed using the smooth Particle Mesh Ewald method [51] with a grid spacing of $1 \AA$.

Subsequent higher temperature simulations were performed sequentially, the last structure from the production run being used as the input for the next equilibration run. A partial set of simulations, performed on cooling, indicated that there was no hysteresis. 


\section{$3 \quad$ Results and Discussion}

\section{$M L V s$}

Figure 3 shows the experimental data for MLVs from D16 and DSC. The isotropic diffraction patterns were radially integrated for every temperature step to result in the color-coded figure of the intensity as a function of the scattering angle $2 \theta$ and temperature $T$. The two phase transitions clearly appear around $13{ }^{\circ} \mathrm{C}(286 \mathrm{~K})$ and $22.5^{\circ} \mathrm{C}(295.5 \mathrm{~K})$ as a Q-displacement of the inter-lamellar Bragg peak. This is in very good agreement with DSC which shows the expected pre-transition and main phase transition at $12.4{ }^{\circ} \mathrm{C}(285.6 \mathrm{~K})$ and $24{ }^{\circ} \mathrm{C}(297.1 \mathrm{~K})$, respectively. From the Bragg peak, a d-spacing of $62.7 \AA$ at $30{ }^{\circ} \mathrm{C}$ in the liquid phase is determined, in accordance with literature values [25]. This d-spacing corresponds to the highest hydration, e.g. more than $40 \%$ weight of water according to Janiak et al. [41], which gives a molar ratio of water $\left(\mathrm{n}_{\mathrm{W}}\right)$ and lipid $\left(\mathrm{n}_{\mathrm{L}}\right)$ of $\mathrm{R}_{\mathrm{W}}=\mathrm{n}_{\mathrm{W}} / \mathrm{n}_{\mathrm{L}}>25[52]$.

EINS data from IN13 are shown in figure 4. Scattered intensities, summed over the whole Qrange are given in figure 4 , and the $\mathrm{MSD}_{\mathrm{GA}}$ and $\mathrm{MSPF}$ are shown in figure 8. The jump between $22{ }^{\circ} \mathrm{C}(295 \mathrm{~K})$ and $27^{\circ} \mathrm{C}(300 \mathrm{~K})$ indicates the main phase transition, but the pretransition does not generate a change in scattering detectable by EINS. The slope of the summed intensities is slightly different before and after the transition since, taking into account the whole Q-range, smaller amplitudes of motion are included which contribute more in the gel and ripple phase than in the fluid phase.

\section{MLBs}

The hydrated, MLB sample was again first characterized by diffraction on D16 (see figure 5). The Bragg peak corresponding to the inter-bilayer distance and its two higher order counterparts, dominate the diffraction pattern. The main transition occurs at about $30{ }^{\circ} \mathrm{C}(303$ $\mathrm{K})$, which is $6{ }^{\circ} \mathrm{C}$ higher than in the MLVs, as expected, since the membranes are less hydrated $[17,41,53]$. 
The d-spacing of $50 \AA$ at $37{ }^{\circ} \mathrm{C}$ (see arrow in figure 5C), corresponding to about 14 water molecules per lipid, allows a comparison with earlier measurements of Janiak et al. [41] indicating a hydration level of about 27 - $28 \%$ weight of water. According to Janiak et al. [41] and Smith et al. [54], DMPC does not reveal the ripple $\mathrm{P}_{\beta}$, phase if the sample is not fully hydrated (below $20 \%$ of water content). The nominal hydration here is slightly higher and figures 5A and 5C indicate indeed a small displacement of the Bragg peak, corresponding to an increase in the d-spacing between 23 and $28^{\circ} \mathrm{C}$, which may be a hint of the pre-transition.

Figure 6 reports the in-plane summed intensity versus T for oriented DMPC MLBs measured on IN6 on heating from 240 to $340 \mathrm{~K}$. No Bragg peaks were observed due to crystalline water at any of the measured temperature points. A 'kink' attributed to the main phase transition temperature occurs close to $30{ }^{\circ} \mathrm{C}(303 \mathrm{~K})$, in good agreement with the diffraction results. On the right scale of figure 6, results from IN13 are reported on dry membranes. No kink or step is visible in the dry sample which remains in the gel phase within the investigated temperature range. For a dry sample, the transition is indeed expected at much higher temperature (around $\left.84{ }^{\circ} \mathrm{C}-357 \mathrm{~K}\right)$ [55], which could not be reached with the experimental setup. Thus the structural rearrangement around the gel-liquid transition is accompanied by a step change in the amplitude of motion revealing the correlation between structure and dynamics.

\section{Molecular dynamics simulations}

MD simulations for the three levels of hydration are analyzed for comparison with the experimental data. The membrane model hydrated with 12 molecules of water per lipid is matched with the MLB data measured on IN6, while the membrane model hydrated with 25 molecules of water per lipid is compared with the MLV data measured on IN13.

As has been done in similar simulation work [50], structural features of the simulated models were tested first. The area per lipid is shown in figure 7 as a function of temperature for the dry and hydrated membranes. Structure snapshots from the gel and liquid phases are shown in figure 2. For all three hydration states the gel phase is equilibrated at $280 \mathrm{~K}$ and in reasonably good agreement with the known experimental value of $48 \AA^{2}$ for the area per lipid [39]. For the dry membrane, increasing temperature causes a slight decrease in the area per lipid reaching a minimum at $320 \mathrm{~K}$, suggesting that this system with very little water actually takes several $100 \mathrm{~ns}$ to fully equilibrate. An increase in area per lipid is observed at $350 \mathrm{~K}$. In 
contrast, the hydrated membrane shows a marked increase in the area per lipid, to approximately the experimental value of $60.6 \AA^{2}$ [25] around $300 \mathrm{~K}$, which is therefore the gel-liquid transition temperature in these simulations. Thereafter, thermal motion causes a progressive expansion in the plane of the liquid membrane. As the system is isolated from the environment in both the simulation and the experiment, in-plane expansion results in thinning perpendicular to the plane together with a chain density decrease of about $2 \%$ [22].

The order parameter $\mathrm{S}_{\mathrm{CH}},\left(\frac{1}{2}\left\langle 3 \cos ^{2} \theta-1\right\rangle\right)$ where $\theta$ is the angle between $\mathrm{CH}$ bonds and a reference direction, here the $\mathrm{z}$-direction, for $\mathrm{C}$ atoms along the tails has also been calculated for hydrated membranes. $\mathrm{S}_{\mathrm{CH}}$ describes how much the DMPC tails are extended and its dependence on $\mathrm{CH}_{2}$ group and temperature agrees well with the NMR measurements (see ESI, figure 2).

Simulation and experiment are also consistent in terms of the dynamical transition, corresponding to local motions of sub-macromolecular groups, as evidenced by EINS. MSDs have been calculated for time intervals of $\delta$ from the MD trajectories through

$$
\operatorname{MSD}(\delta t)=\left\langle|\vec{r}(t+\delta t)-\vec{r}(t)|^{2}\right\rangle
$$

where $\vec{r}(t)$ is the position of an atom at time $t$ and $\vec{r}(t+\delta t)$ at a time $\delta t$ later. For MLV's, the MSD from the membrane model hydrated with 25 water molecules per lipid at 100ps, corresponding to the resolution of IN13 (see ESI, figure 3), is plotted in figure 8 along with the $\mathrm{MSD}_{\mathrm{GA}}$ and MSPF extracted from the corresponding experimental data. In this case, the experimentally determined $\mathrm{MSD}_{\mathrm{GA}}$ is significantly smaller than that obtained from simulations. However the MSPF is about twice as big as the experimental $\mathrm{MSD}_{\mathrm{GA}}$ across the whole temperature range and is in better agreement with the MSD from simulations.

For MLBs, the MSD from the membrane model hydrated with 12 water molecules per lipid at $10 \mathrm{ps}$, corresponding to the resolution of IN6, is plotted in figure 9 along with the $\mathrm{MSD}_{\mathrm{GA}}$ and MSPF extracted from the corresponding experimental data. All data show a step in the dynamical amplitude at approximately $300 \mathrm{~K}$. The experimentally determined $\mathrm{MSD}_{\mathrm{GA}}$ is in quantitatively good agreement with that obtained from MD simulations, whereas the experimental MSPF is approximately $40 \%$ higher above $300 \mathrm{~K}$. 
Figures 7, 8 and 9 show that the temperature of the gel-liquid phase transition is wellreproduced by the MD simulations of the hydrated membranes and that the dynamical amplitudes obtained from simulations are also in good agreement (within a factor of 1.5) with experimental values, given that these vary by a factor $\sim 2$ depending on the analysis method. This level of agreement for dynamical amplitudes is comparable to that obtained by us in previous work on myoglobin [56] between the MSD from MD simulations and the experimentally-determined $\mathrm{MSD}_{\mathrm{GA}}$.

Simulations offer the possibility to discriminate between parts of the lipid. Figures 8 and 9 also show the contribution to the total MSD from the lipid head group and tails. The tails have a slightly higher MSD than the head group and, in particular, the tails show clearly the dynamic transition whereas for the head groups it is much less pronounced.

Finally, figure 10 shows the projection of the MSD for the hydrated membrane in the plane of the membrane (XY) and in the perpendicular direction $(\mathrm{Z})$. The trend in the calculated data the amplitude of in-plane motion is bigger than that in the perpendicular direction - has also been observed experimentally [57]. However the anisotropy is slightly more pronounced in the calculations (about a factor of 1.5) compared to the experiment (about 30\%). The angular integration in the experiment which partially averages over a range of membrane orientations contributes to this discrepancy.

\section{Discussion and Conclusions}

Diffraction (and calorimetry in the case of the MLVs) shows the effect on the main gel-liquid phase transition of membrane geometry. For MLVs this occurs at $23 \pm 1{ }^{\circ} \mathrm{C}(296 \pm 1 \mathrm{~K})$ while for MLBs (about 14 water molecules per lipid) the transition temperature is shifted about $7{ }^{\circ} \mathrm{C}$ higher to $30 \pm 2{ }^{\circ} \mathrm{C}(303 \pm 2 \mathrm{~K})$. EINS data shows that molecular motions, on the microscopic length-scale of several $\AA$ and the time-scale of ps-ns, change at approximately the same temperature as observed by diffraction and calorimetry. For MLVs, the temperature resolution of the EINS experiment was $2.5^{\circ} \mathrm{C}$ and the dynamical transition was observed between $25^{\circ} \mathrm{C}$ and $27.5{ }^{\circ} \mathrm{C}$, slightly higher than for the structural transition. For MLBs, the dynamical transition is observed at $29 \pm 2{ }^{\circ} \mathrm{C}(302 \pm 2 \mathrm{~K})$ matching well the structural transition 
temperature. A discrepancy of a degree or two between different observations of $T_{m}$ is assigned to the fact that one diffractometer and two spectrometers were used with their specific sample environments. The effect of hydration has been investigated for the MLBs, a dry sample not showing any evidence of a dynamical phase transition up to $57^{\circ} \mathrm{C}(330 \mathrm{~K})$.

MD simulations show for the hydrated MLBs, through and above $\mathrm{T}_{\mathrm{m}}$, that the MSD increases significantly and that the microscopic dynamical and structural transitions, as shown by the MSD and the surface area per lipid respectively, are perfectly concurrent. The temperature precision in the $\mathrm{MD}$ study is $10^{\circ} \mathrm{C}$ and the main transition is observed above $17^{\circ} \mathrm{C}(290 \mathrm{~K})$ and by $27{ }^{\circ} \mathrm{C}(300 \mathrm{~K})$, for 12 water molecules per lipid, in good agreement with experiment $30 \pm 2{ }^{\circ} \mathrm{C}$ for MLBs. For 25 molecules per lipid, the transition occurs above $27{ }^{\circ} \mathrm{C}(300 \mathrm{~K})$ and by $37{ }^{\circ} \mathrm{C}(310 \mathrm{~K})$, compared to $25 \pm 2{ }^{\circ} \mathrm{C}$ for MLVs. Given the 10 degree temperature interval between MD runs, the maximum discrepancy between the simulations of these two hydration levels is 20 degrees and the minimum discrepancy is 0 degrees. The transition temperature for these two levels of hydration is expected to be the same [41] and a smaller temperature difference between MD runs would allow this to be verified. As in the experiment, MD simulations show that dry membranes remain in the gel phase up to $57^{\circ} \mathrm{C}$, after which there is an up-turn in the surface area per lipid (figure 7) and in the MSD (figure 9).

MD simulations accurately reproduce structural parameters like the surface area per lipid (figure 7) and the order parameter (ESI, figure 2), which describes the folding of chains, for MLBs. Some of this data (surface area per lipid) is actually used to refine the force field, in which case the agreement simply validates the simulation protocol used here. Interestingly, the dynamical description of the lipids is in generally good agreement with that obtained from experiments. Dynamical amplitudes have been derived experimentally with two methods, the Gaussian approximation $\left(\mathrm{MSD}_{\mathrm{GA}}\right)$ which only exploits low $\mathrm{Q}$ data and the MSPF which uses the whole $\mathrm{Q}$ range of data. The difference between these two analyses is about a factor of 2 , the MSPF being bigger than the $\mathrm{MSD}_{\mathrm{GA}}$ from the Gaussian approximation. In principle the MSPF should give a more accurate value for the dynamical amplitude, to be compared with the MSD from simulations which is free of any approximations and effectively integrates over the whole Q range. In the case of MLVs (figure 8), the MSPF is about 20\% lower than the MSD from MD simulations of the highest hydrated membrane. For MLBs, the MSPF is about 
$40 \%$ higher than the MSD from simulations. In terms of dynamical amplitudes $(\sqrt{ } \mathrm{MSD}$ or $\sqrt{\text { MSPF}}$, the agreement between experiment and simulation is therefore within $20 \%$. Simulations show that the dynamical changes occur mainly for the lipid tails in the membrane plane (figures 8 and 9).

Given this level of agreement between experiment and simulation that have been performed concurrently, the dynamical features of the atomistic simulation could reliably be used to build an analytical model of lipid dynamics in membranes to analyze more extensive, quasielastic neutron scattering data and therefore establish a coarse-grained, theoretical description of membrane dynamics and phase behavior based on molecular dynamics. The need for a realistic, physical model of membrane dynamics to analyze experimental data is highlighted here by the good but certainly not perfect agreement between experiment and simulation, so experiment has a key role to play in determining dynamical amplitudes and time-scales, based on an appropriate physical model.

Finally the agreement between simulations and experiment analyzed with different methods is now close enough to motivate a systematic, coherent study of the available, large body of experimental and simulation data. The goal will be to determine the merits and limitations of experiment analysis methods and the most accurate way to compare with simulation data, This will, for example, involve extending the MSD-based analysis of simulations used here, with a time cut-off corresponding to the instrument resolution, to the full calculation of neutron scattering spectra, including their convolution with the precise instrument resolution function, which can then be treated in exactly the same way as experimental spectra.

\section{Acknowledgements}

We are grateful to ILL for the allocation of beam-time.

\section{References}

1. O. Mouritsen, Life as a Matter of Fat (Springer, 2005).

2. http://www.ucalgary.ca/tieleman/?page=Peter_Tieleman

3. http://md.chem.rug.nl/ marrink/science.html

4. http://www.memphys.sdu.dk/people/personal pages/ogm/

5. J.S. Hub, T. Salditt, M.C. Rheinstädter, and B.L. de Groot, Biophys. J., 93, 3156-3168 (2007).

6. C. F. Majkrzak, N. F. Berk, S. Krueger, J. A. Dura, M. Tarek, D. Tobias, V. Silin, C. W. Meuse, J. Woodward, and A. L. Plant, Biophys. J., 79, 3330-3340 (2000).

7. M. Tarek, D. J. Tobias, S.-H. Chen, and M. L. Klein, Phys. Rev. Lett., 87, 238101, 1 - 4 (2001). 
8. T. Heimburg, Biophysical Journal, 78, 1154 (2000).

9. J.R. Trudell, D.G. Payan, J.H. Chin, and E.N. Cohen, Proceedings of the National Academy of Sciences, 72, 210-213 (1975).

10. A. Macdonald, Biochimica et Biophysica Acta - Biomembranes, 507, 26-37 (1978).

11. J. Nagle, and D. Wilkinson, Biophysical Journal, 23, 159-175 (1978).

12. P. Brocca, L. Cantu, M. Corti, E. Del Favero, S. Motta, and M.C. Nodari, Colloids Surf. A., 291, $63-68$ (2006).

13. H. Ichimori, T. Hata, H. Matsuki, and S. Kaneshina, Biochimica et Biophysica Acta Biomembranes, 1414, 165-174 (1998).

14. S. Krishna Prasad, R. Shashidhar, B. Gaber, and S. Chandrasekhar, Chemistry and Physics of Lipids, 43, 227-235 (1987).

15. H.H. Mantsch, and R.N. McElhaney. Chem Phys Lipids, 57, 213-226 (1991).

16. R. Winter, and W. Dzwolak, Philosophical Transactions of the Royal Society A: Mathematical, Physical and Engineering Sciences, 363, 537-563 (2005).

17. R. Winter, and C. Jeworrek, Soft Matter, 5, 3157-3173 (2009).

18. C. Sennoga, A. Heron, J. M. Seddon, R. H. Templer and B. Hankamer, Acta Cryst. D, 59, 239 246 (2003).

19. T. Salditt, C. Münster, U. Mennicke, C. Ollinger and G. Fragneto, Langmuir, 19, 7703 - 7711 (2003).

20. H.P. Wacklin, Curr. Op. in Coll. Interf. Sc., 15, 445 - 454 (2010).

21. E. Dufourc, NMR of Lipids, Wiley Encyclopedia of chemical biology, John Wiley \& Son, 2008.

22. J. Phillips et al, J Comput Chem, 26, 1781 (2005).

23. J. B. Klauda et al, J. Phys. Chem. B, 114, 7830-7843 (2010).

24. L. Rusevich et al., Eur. Phys. J. E, $36: 80,1-9$ (2013).

25. N. Kucerka, Y. Liu, N. Chu, H.I. Petrache, S. Tristram-Nagle, and J. F. Nagle, Biophys. J., 88, 2626-2637 (2005).

26. C. L. Armstrong, M.A. Barrett, L. Toppozini, N. Kucerka, Z. Yamani, J. Katsaras, G. Fragneto, and M. C. Rheinstädter, Soft Matter, 8, 4687-4694 (2012).

27. A. Blume, Biochem., 22, 5436-5442 (1983).

28. S. Leekumjorn, and A.K. Sum, BBA, 1768, $354-365$ (2007).

29. M. Trapp, T. Gutberlet, F. Juranyi, T. Unruh, B. Demé, M. Tehei and J. Peters, J. Chem Phys, 133, 164505, 1-7 (2010).

30. P. Brocca, L. Cantù , M. Corti, E. Del Favero, S. Motta, M.C. Nodari. Colloids and Surfaces A: Physicochem. Eng. Aspects, 291, 63-68 (2006).

31. K. Lohner, E. Sevcsik and G. Pabst, Advances in planar lipid bilayers and liposomes, volume 6, (Elsevier, 2008).

32. W. Pfeiffer, T. Henkel, E. Sackmann, W. Knoll, and D. Richter, Europhys. Lett., 8, 201-206 (1989).

33. S. König, W. Pfeiffer, T. Bayerl, D. Richter and E. Sackmann, J. Phys. II, 2, 1589 - 1615 (1992).

34. S. König, E. Sackmann, D. Richter, R. Zorn, C. Carlile, and T. M. Bayerl. The Journal of Chemical Physics, 100, 3307-3316 (1994).

35. E. Falck, T. Rog, M. Karttunen and I. Vattulainen, J. Am. Chem. Soc., 130, 44 - 45 (2008).

36. S. Busch, C. Smuda, L. C. Pardo, and T. Unruh, J. Am. Chem. Soc., 132, 3232-3233 (2010).

37. G.R. Kneller, K. Baczynski, and M. Pasenkiewicz-Gierula, J. Chem. Phys., 135, 141105, 1 - 4 (2011).

38. C.L. Armstrong, M. Trapp, J. Peters, T. Seydel, and M.C. Rheinstädter, Soft Matter, 7, 8358-8362 (2011).

39. S. Tristram-Nagle, Y. Liu, J. Legleiter and John F. Nagle, Biophys. J., 83, 3324-35 (2002).

40. http://www.ill.eu/instruments-support/instruments-groups/instruments/d16/characteristics/

41. M. J. Janiak, D. M. Small, G. G. Shipley, J. Biol. Chem., 254, 6068-6078 (1979).

42. F. Natali, J. Peters, D. Russo, S. Barbieri, C. Chiapponi, A. Cupane, A. Deriu, M.T. Di Bari, E. Farhi, Y. Gerelli, P. Mariani, A. Paciaroni, C. Rivessau, G. Schiro', F. Sonvico, Neutron News, 19, 14 (2008). 
43. http://www.ill.eu/instruments-support/instruments-groups/instruments/in6/description/instrumentlayout/

44. A. Rahman, K. S. Singwi, and A. Sjölander, Phys Rev, 126, 986-996 (1962).

45. M. Tehei, R. Daniel and G. Zaccai, Eur. Biophys. J. 35, 551 (2006).

46. F. Natali, C. Castellano, D. Pozzi, and A. Congiu Castellano, Biophys. J. 88, 1081 (2005).

47. J. Peters, and G.R. Kneller, J. Chem. Phys., 139, 165102, 1 - 5 (2013).

48. http://www.charmm-gui.org/

49. R.W. Pastor, R.M. Venable, and S.E. Feller, Acc. Chem. Res., 35, 438 - 446 (2002).

50. C.-J. Högberg, and A.P. Lyubartsev, J. Phys. Chem. B. 110, 14326 - 14336 (2006).

51. U. Essmann et al, J. Chem. Phys., 103, 8577 (1995).

52. H. Pfeiffer, H. Binder, G. Klose, and K. Heremans, Biochim. Biophys. Acta 1609, 148 - 152 (2003).

53. M. Trapp, F. Juranyi, M. Tehei, L. van Eijck, B. Demé, T. Gutberlet and J. Peters, Spectroscopy 24, 461 - 466 (2010).

54. G. S. Smith, E. B. Sirota, C. R. Safinya, and N. A. Clark, Phys. Rev. Lett. 60, 813-816 (1988).

55. A.V. Popova, and D.K. Hincha, BMC Biophysics, 2011, 4, 11.

56. A.M. Stadler, E. Pellegrini, M. Johnson, J. Fitter, and G. Zaccai, Biophys. J. 102, $351-359$ (2012).

57. F. Natali, A. Relini, A. Gliozzi, R. Rolandi, P. Cavatorta, A. Deriu, A. Fasano, and P. Riccio, Chem. Phys., 292, 455-464 (2003). 


\section{Figure and table captions}

Figure 1: Lipid polymorphism: MLV and MLB.

Figure 2: Schematic representation of the different lipid phases of DMPC at full hydration.

Figure 3: Color-coded intensities of DMPC MLVs as a function of the scattering vector Q and temperature $\mathrm{T}$. The 001 reflection of the three distinct phases is clearly visible while a weak signal only visible in the $\mathrm{P}_{\beta}$ ' phase corresponds to the in-plane period of the ripples. The white line represents a DSC measurement, reported vertically as function of temperature.

Figure 4: Summed elastic intensity of DMPC MLVs as a function of temperature. The lines are guides to the eyes and the dashed line indicates the phase transition temperature from diffraction (figure 3).

Figure 5: A - Color-coded intensities of DMPC MLBs as a function of momentum transfer Q and temperature T. First, second and third order Bragg peaks are clearly visible. B Intensities as function of $\mathrm{Q}$ in the phases below and above the main transition. $\mathrm{C}-$ Extracted lamellar d-spacing as function of temperature. The dashed line marks the main phase transition and the red arrow indicates the point which was used to determine the hydration level.

Figure 6: In-plane, summed elastic intensities versus T for the DMPC MLBs (IN6). The lines are guides to the eyes and the dashed line indicates the phase transition temperature from diffraction (figure 5). In comparison IN13 in-plane summed intensities are shown for dry membranes on the right scale.

Figure 7: Surface area per lipid as a function of temperature for three hydration states: dry membrane (red triangles) and membrane hydrated at 12 and 25 water molecules per lipid (full and hollow blue squares). Error bars, derived from simulation cell fluctuations, are typically \pm $1 \AA^{2}$. Experimental values for the gel and liquid phases, as given in the text, are also indicated as stars for the liquid and gel phases, respectively. The dashed vertical line indicates the measured phase transition temperature.

Figure 8: MSD of MLVs as a function of temperature and hydration obtained from data simulated for the $100 \mathrm{ps}$ time interval and measured on IN13. Simulated data are in blue. Experimental results were extracted as MSPF (in red) and $\mathrm{MSD}_{\mathrm{GA}}$ (in black) and both are shown for comparison. The contributions of the head group and tails of hydrated DMPC are also shown. The dashed vertical line indicates the phase transition temperature.

Figure 9: MSD of MLBs from MD simulations taken for the $10 \mathrm{ps}$ time interval compared to $\mathrm{MSD}_{\mathrm{GA}}$ and MSPF data from IN6 as a function of temperature. Simulated data are in blue. The MSPF is in red. The experimental $\mathrm{MSD}_{\mathrm{GA}}$ data (in black) were rebinned in order to reduce the statistical variation. The contributions of the head group and tails of the MLBs are also shown. The dashed vertical line indicates the phase transition temperature.

Figure 10: MSD from MD simulations for the $100 \mathrm{ps}$ time window as a function of temperature for the hydrated membrane (at 12 and 25 water molecules per lipid) in the plane of the membrane (XY) and in the perpendicular direction $(\mathrm{Z})$.

Table 1: Characteristics of the spectrometers at ILL used in this study. 


\section{Figures}

Figure 1:
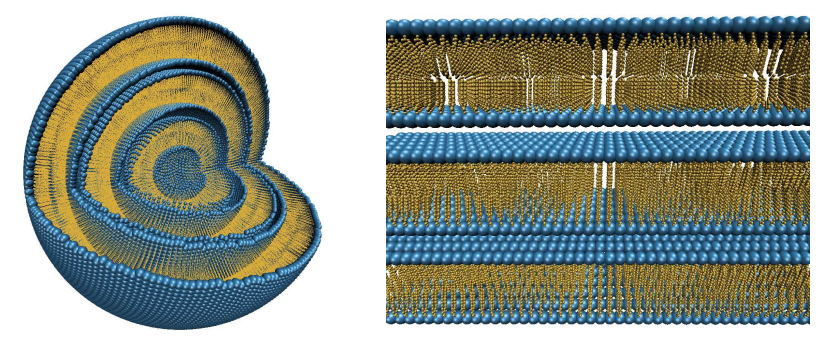

Figure 2:

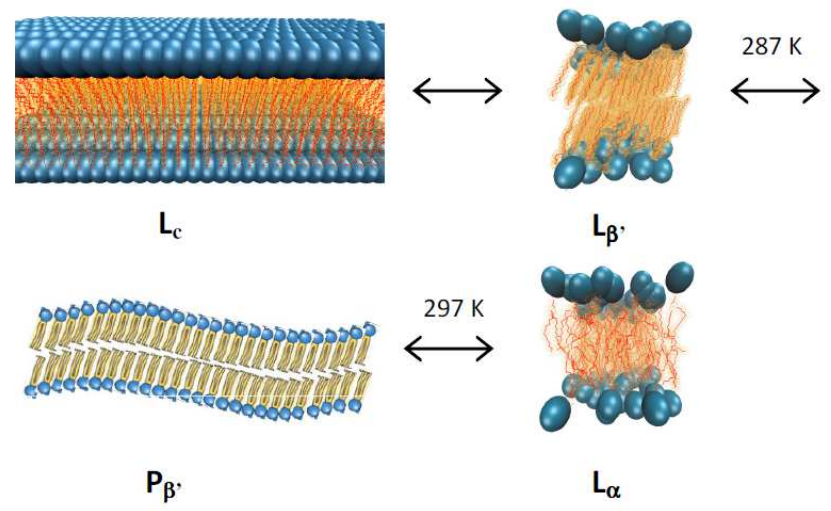

Figure 3 :

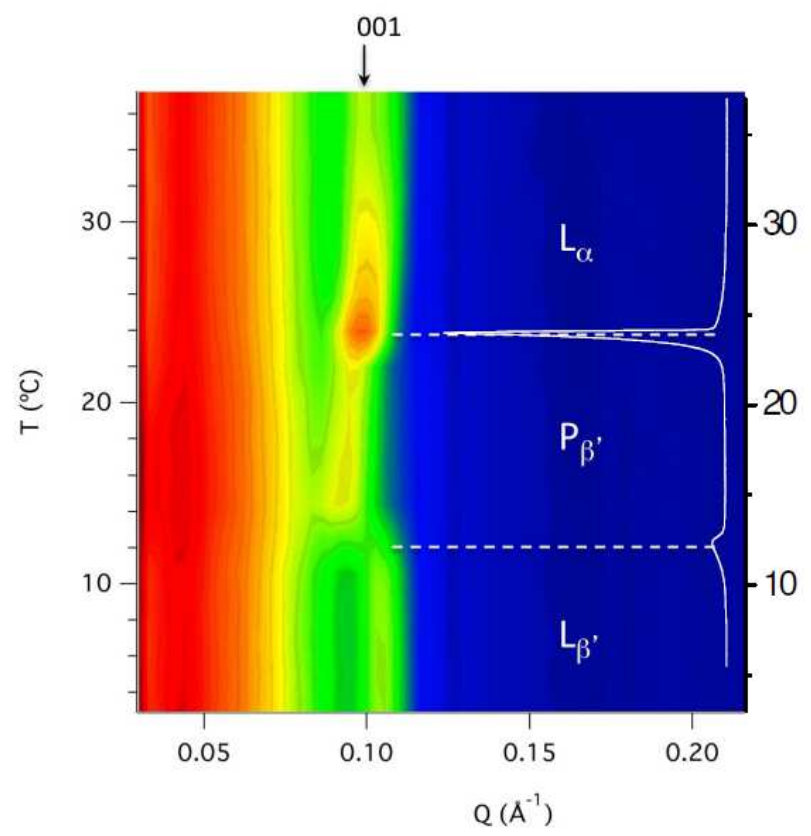


Figure 4:

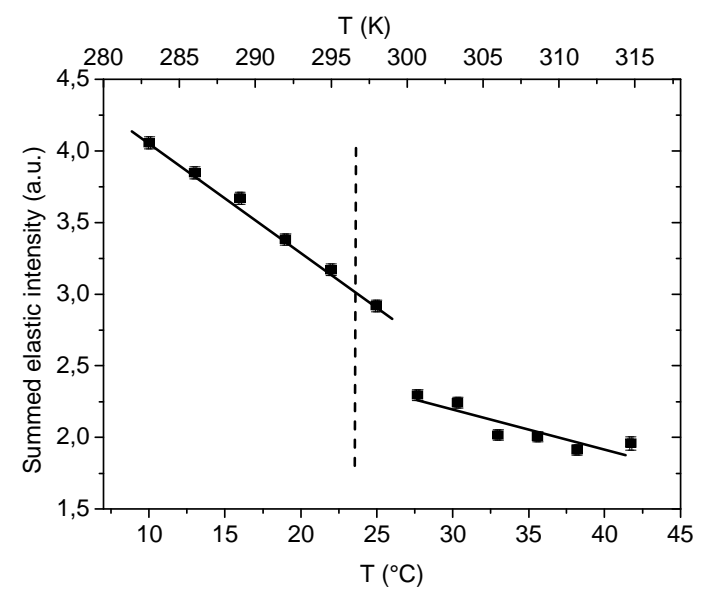

Figure 5:
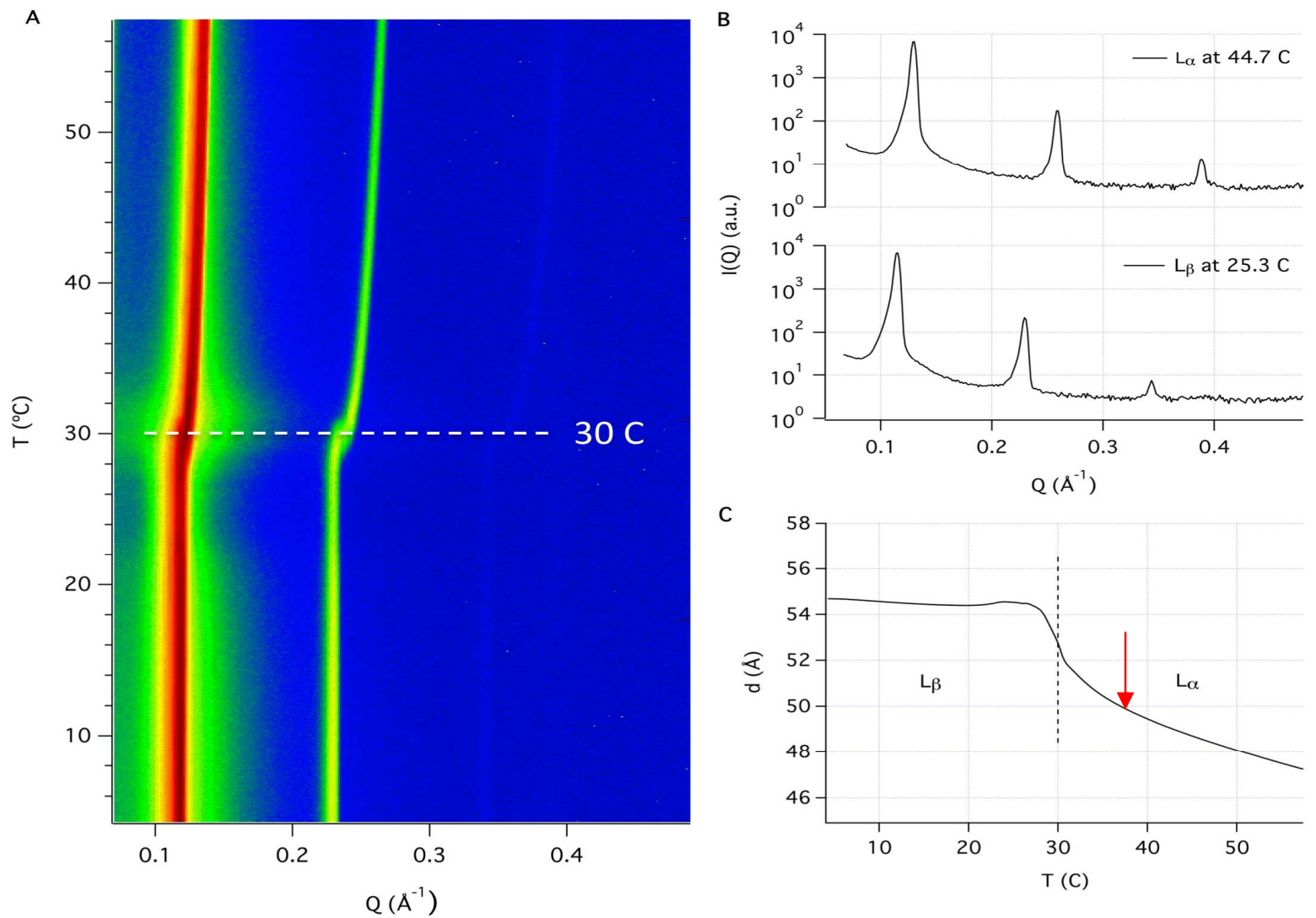
Figure 6:

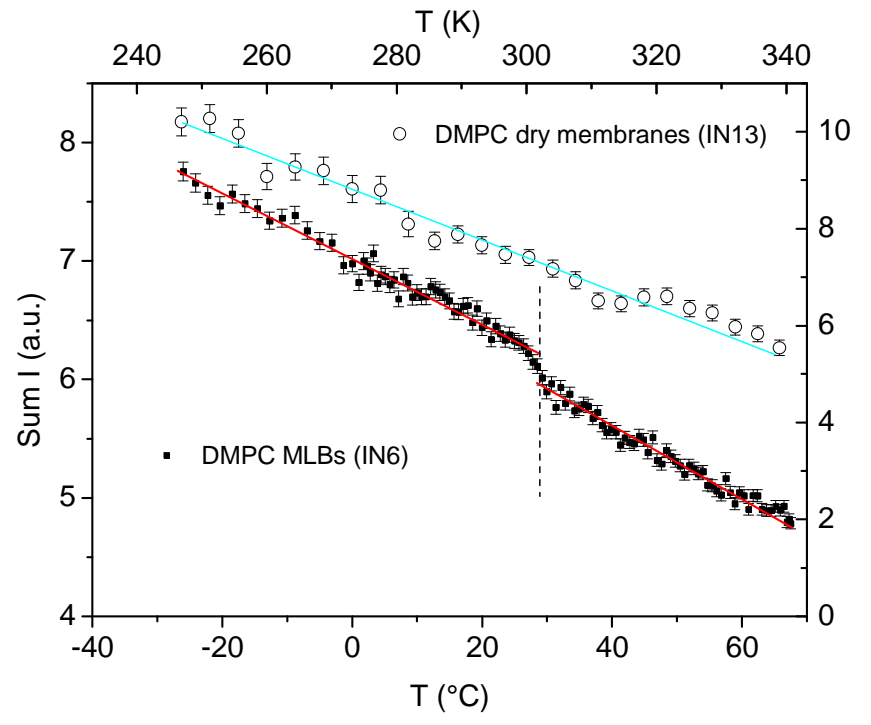

Figure 7:

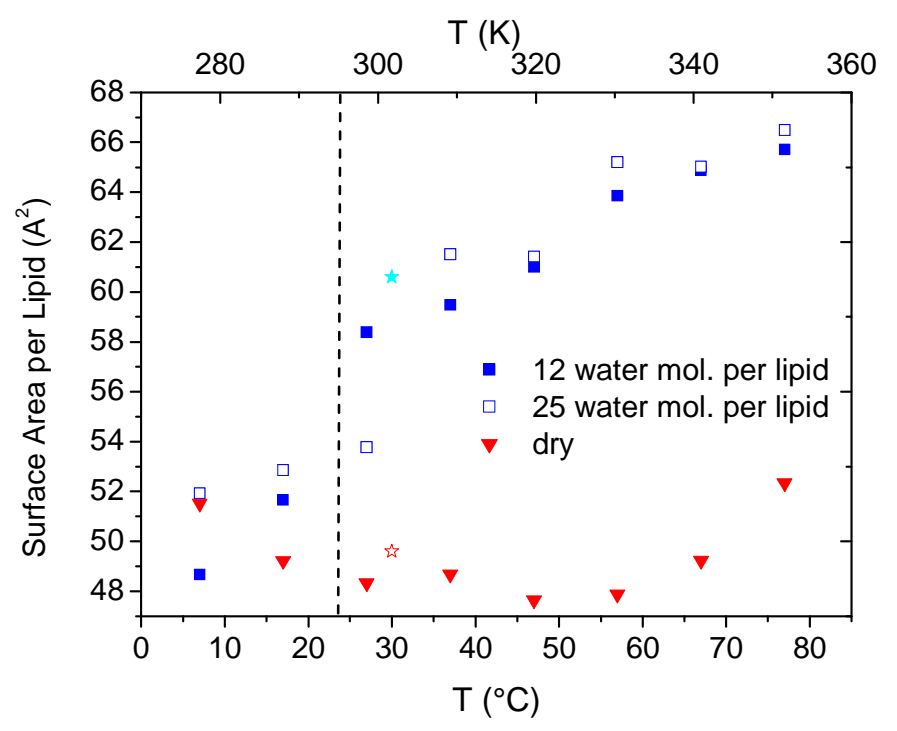


Figure 8 :

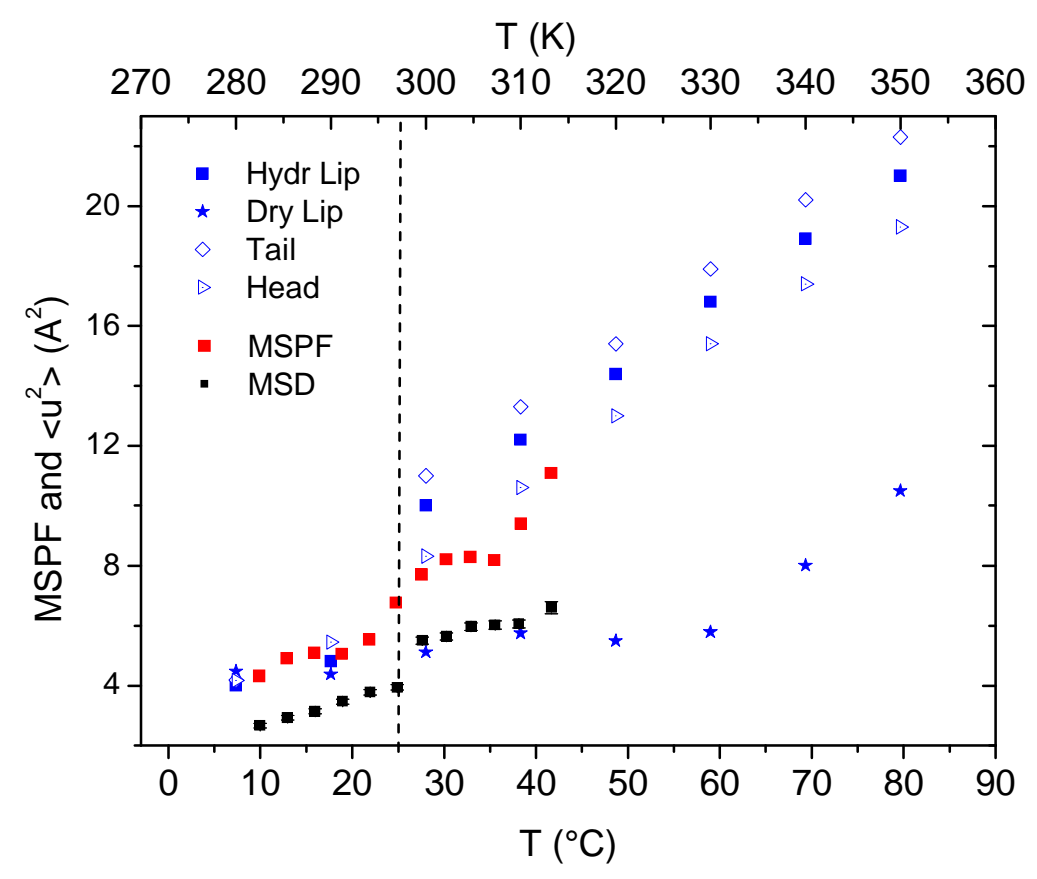

Figure 9:

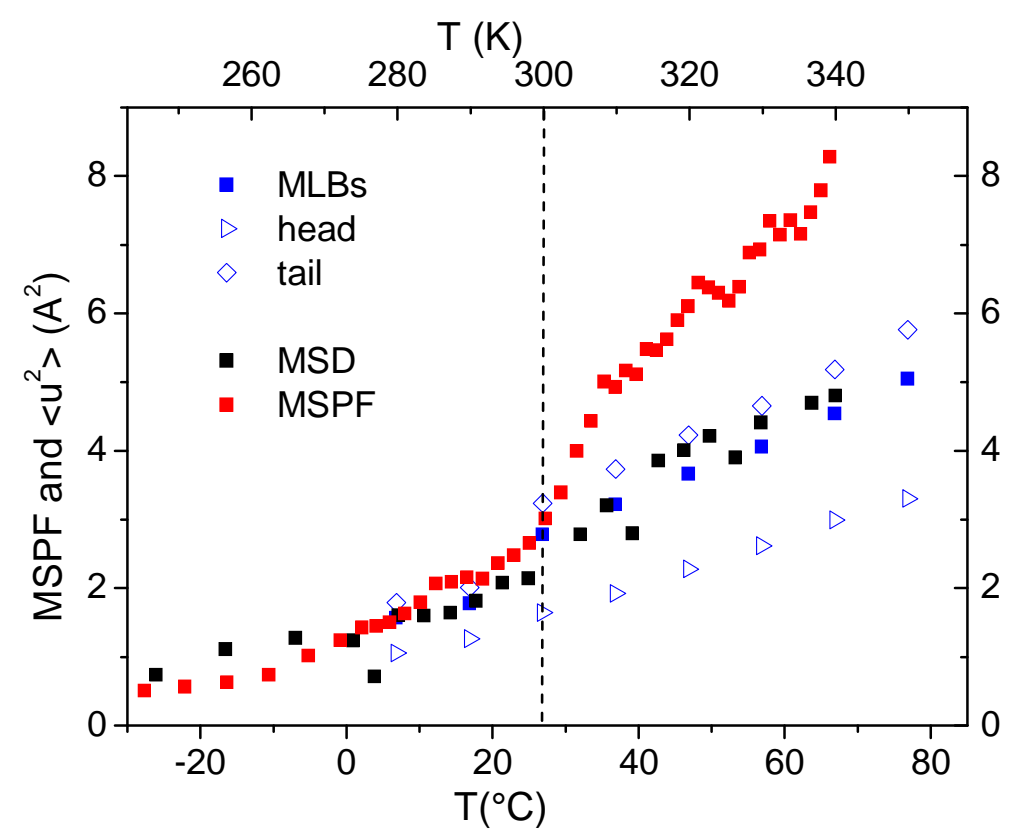


Figure 10:

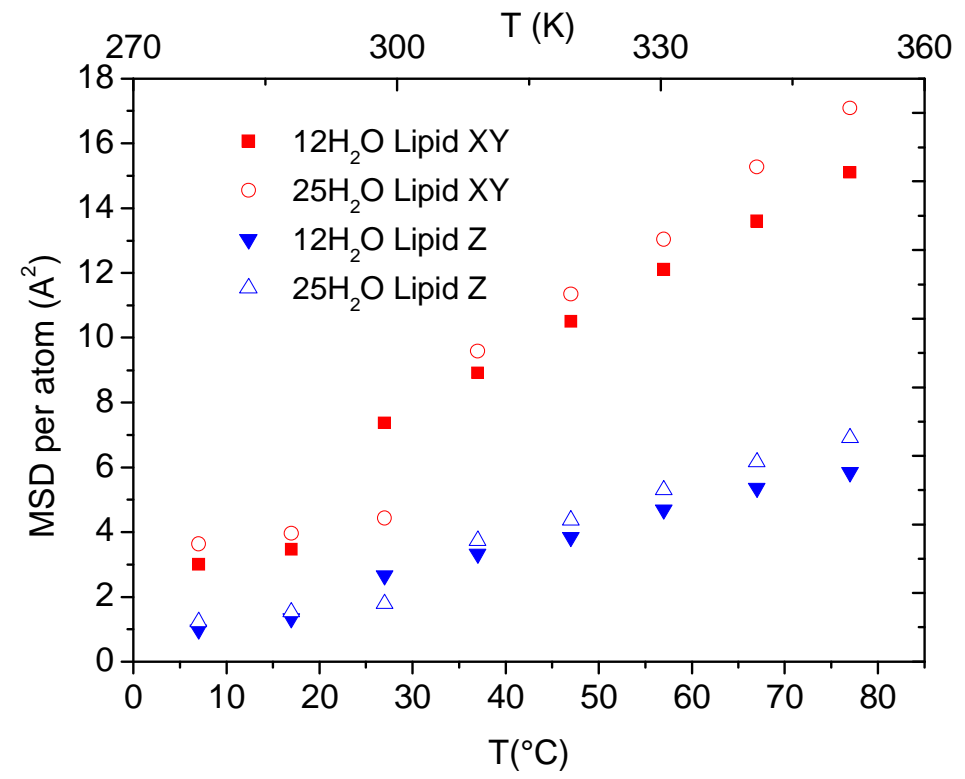

Table 1:

\begin{tabular}{|c|c|c|}
\hline Spectrometer & IN6 & IN13 \\
\hline Wavelength $[\check{A}]$ & 5.1 & 2.23 \\
\hline Accessible Q-range $\left[\AA^{-1}\right]$ & $0.4-2.0$ & $0.2-4.9$ \\
\hline Length-scale $[\AA]$ & $3-16$ & $1-6$ \\
\hline Resolution FWHM $[\mu \mathrm{eV}]$ & 90 & 8 \\
\hline Time-scale [ps] & 10 & 100 \\
\hline
\end{tabular}




\title{
Electronic Supporting Information (ESI)
}

\author{
Aoun et al.
}
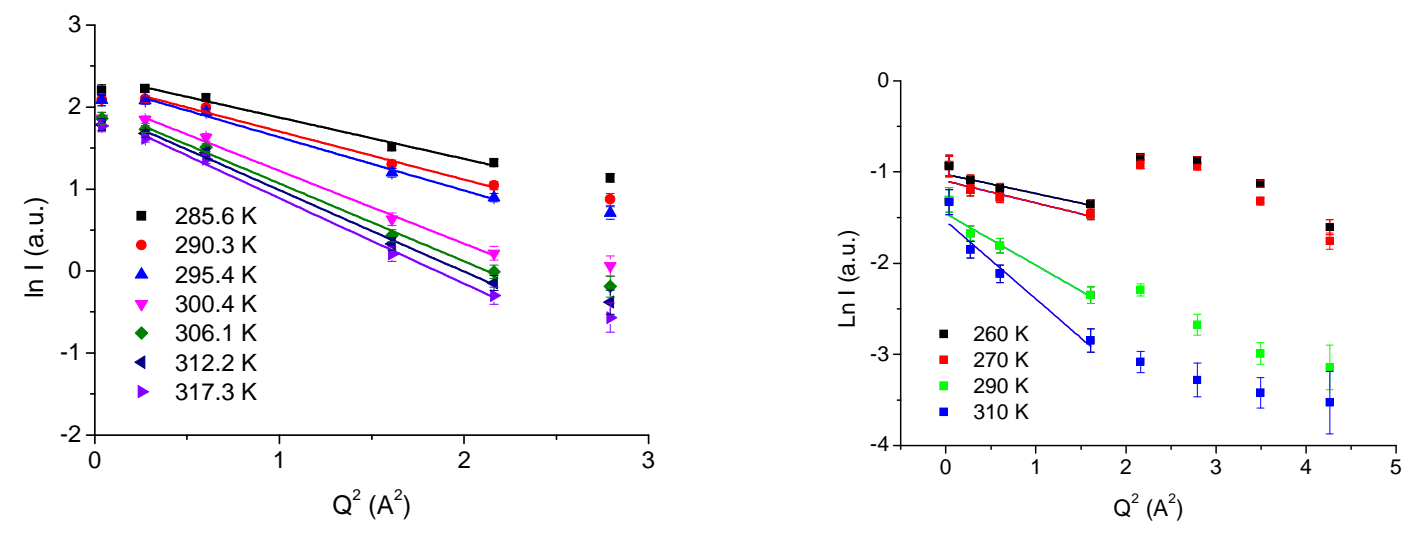

Figure 1: Fits of the $\ln \mathrm{I}_{\mathrm{el}}(\mathrm{Q})$ versus $\mathrm{Q}^{2}$ in case of MLVs (left) and MLBs (right) measured on IN13. Whereas the curves are nicely linear in case of MLVs, the coherent chain correlation peak is clearly visible for MLBs below the gel transition. Therefore we fitted only points below the corresponding Q-value. 


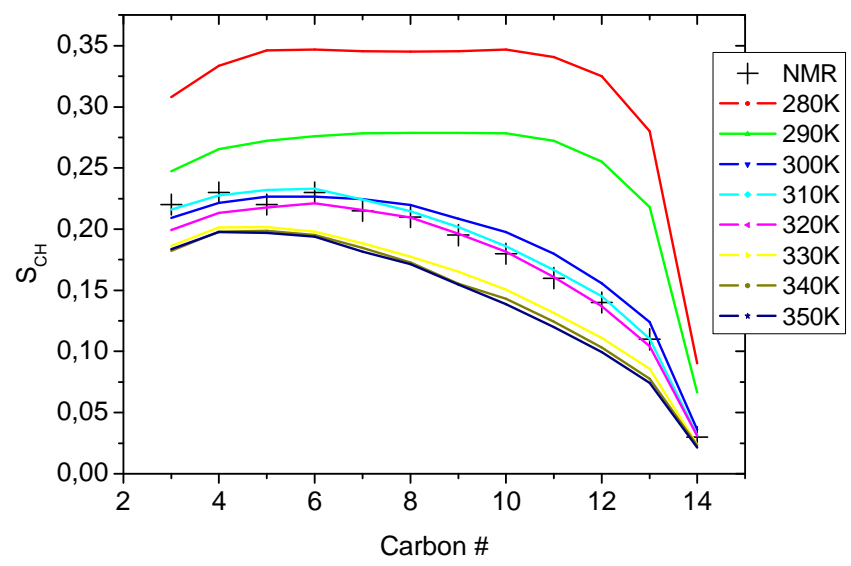

Figure 2: The order parameter $\mathrm{S}_{\mathrm{CH}}$ as a function of $\mathrm{C}$ atom in the hydrated membrane DMPC tails and as a function of temperature. Experimental values at $300 \mathrm{~K}$ are taken from reference (46). The top two curves ( 280 and $290 \mathrm{~K}$ ) are for the gel phase, whereas all other curves below are in the liquid phase. 
MSDs versus time frames, corresponding to different instrumental energy resolutions: Atomic mean square displacements are calculated by averaging over increasing time frames chosen along the particle trajectories.

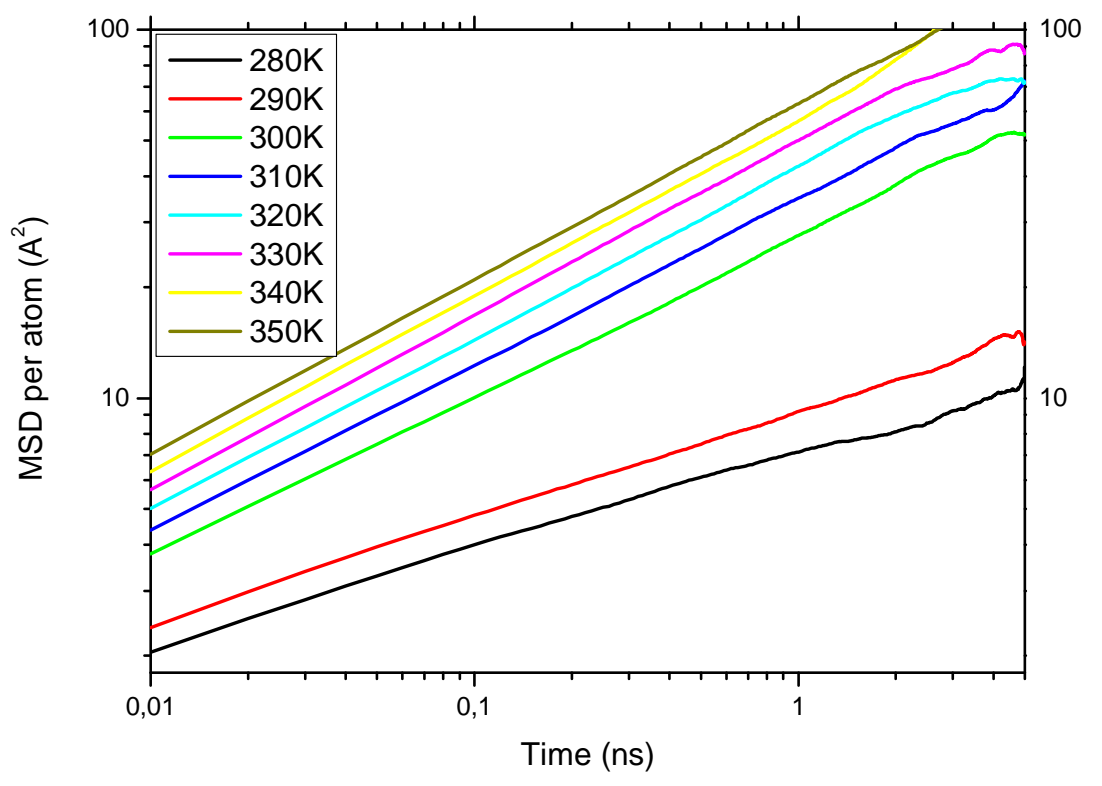

Figure 3: Log-log plot of the MSDs versus time frames calculated along the trajectories of the production run of $5 \mathrm{~ns}$ for the fully hydrated membrane - 12 water molecules per lipid. 